\title{
HERMITIAN $*$-EINSTEIN SURFACES
}

\author{
GEO GRANTCHAROV AND OLEG MUŠKAROV
}

(Communicated by Jonathan M. Rosenberg)

\begin{abstract}
We study the problem when a compact Hermitian $*$-Einstein surface $M$ is Kählerian and show that it is true if $M$ is additionally assumed to be either Einstein or anti-self-dual. We also prove that if the *-scalar curvature of $M$ is positive then $M$ is a conformally Kähler surface with positive first Chern class.
\end{abstract}

\section{INTRODUCTION}

A well-known conjecture of Goldberg [Go] says that any compact almost Kähler Einstein manifold is Kählerian. This conjecture is still open, but some progress has been made under additional curvature conditions [Go, O1, S1, SV]. In particular, Sekigawa and Vanhecke [SV] have proved that the Goldberg conjecture is true for four-dimensional *-Einstein manifolds with constant $*$ scalar curvature.

The purpose of this note is to obtain Hermitian analogs of the abovementioned result. More specifically, we study the problem when a compact Hermitian *-Einstein surface is Kählerian. Our interest in this problem was also motivated by the following observations. First, any compact Hermitian *Einstein surface with positive $*$-scalar curvature is globally conformally Kähler (cf. Theorem 3.1). Second, the Page metric on the complex surface $F_{1}$ [B, 11.82 ] is Einstein $*$-Einstein and with nonconstant positive $*$-scalar curvature. Since the Page metric is non-Kähler, the following conjecture seems to be true: Any compact Hermitian $*$-Einstein surface $M$ with constant positive $*$-scalar curvature is Kählerian.

In this note we prove the above conjecture under the additional assumption that $M$ is either Einstein or anti-self-dual (Corollaries 4.2 and 4.3). These results are consequences of the following

Theorem 1.1. Let $M$ be a compact Hermitian Einstein and *-Einstein surface. Then either

(i) $M$ is Kählerian, or

(ii) the metric of $M$ is conformal to an extremal Kähler metric with nonconstant positive scalar curvature. Moreover, the scalar curvature of $M$ is a positive constant, and the *-scalar curvature of $M$ is positive and nonconstant.

Received by the editors July 22, 1991.

1991 Mathematics Subject Classification. Primary 53C55, 53C25.

(C) 1993 American Mathematical Society $0002-9939 / 93 \$ 1.00+\$ .25$ per page 
The converse statement is also true.

We should note that the proof of Theorem 1.1 relies heavily on some results of Derdzinski [D], Vaisman [V], Sekigawa [S2], and Kodâ [K].

\section{Preliminaries}

Let $M=(M, J, g)$ be a Hermitian surface (i.e., a Hermitian manifold of real dimension four) with complex structure $J$ and compatible Riemannian metric $g$. Denote by $\Omega$ the Kähler form of $M$ given by $\Omega(X, Y)=g(X, J Y)$ for $X, Y \in \chi(M)$, where $\chi(M)$ is the Lie algebra of all smooth vector fields on $M$. We assume that $M$ is oriented by the volume form $d M=\frac{1}{2} \Omega^{2}$. It is well known [V] that $d \Omega=\omega \wedge \Omega$, where $\omega=\delta \Omega \circ J$ is the Lee form of $M$. Recall [V] that $M$ is called locally conformally Kähler provided $d \omega=0$.

Let $\nabla, R, \rho$, and $\tau$ be the Riemannian connection, the Riemannian curvature tensor, the Ricci tensor, and the scalar curvature of $M$, respectively. Here

$$
\begin{gathered}
R(X, Y, Z, W)=g\left(\left[\nabla_{X}, \nabla_{Y}\right] Z-\nabla_{[X, Y]} Z, W\right), \\
\rho(X, Y)=\sum_{s} R\left(E_{s}, X, Y, E_{s}\right), \quad \tau=\sum_{s} \rho\left(E_{s}, E_{s}\right)
\end{gathered}
$$

for $X, Y, Z, W \in \chi(M)$, where $\left\{E_{s}\right\}$, is an orthonormal frame. Furthermore we denote by $\rho^{*}$ and $\tau^{*}$ the $*$-Ricci tensor and the $*$-scalar curvature of $M$ defined respectively by

$$
\rho^{*}(X, Y)=\sum_{s} R\left(E_{s}, X, J Y, J E_{s}\right), \quad \tau^{*}=\sum_{s} \rho^{*}\left(E_{s}, E_{s}\right) .
$$

By using the first Bianchi identity we get

$$
\rho^{*}(X, Y)=-\frac{1}{2} \sum_{s} R\left(X, J Y, E_{s}, J E_{s}\right) .
$$

Note that on a Kähler manifold the $*$-Ricci tensor and the Ricci tensor coincide. We shall say that $M$ is a $*$-Einstein surface if the $*$-Ricci tensor is a functional multiple of the metric $g$, i.e., if $\rho^{*}=\tau^{*} / 4 \cdot g$. Note that in contrast to Einstein manifolds the $*$-scalar curvature of a $*$-Einstein surface need not be a constant. On any Hermitian surface the Ricci tensor and the $*$-Ricci tensor are related by

$$
\rho(X, Y)+\rho(J X, J Y)-\rho^{*}(X, Y)-\rho^{*}(J X, J Y)=\frac{\left(\tau-\tau^{*}\right)}{2} \cdot g(X, Y)
$$

for $X, Y \in \chi(M)$ (cf., e.g., [TV]). We also have [V]

$$
\tau-\tau^{*}=2 \delta \omega+\|\omega\|^{2}
$$

Denote by $\gamma_{1}$ the generalized first Chern form of $M$ [S2]. Then

$$
8 \pi \gamma_{1}(X, Y)=-\sum_{s}\left(2 R\left(X, Y, E_{s}, J E_{s}\right)+g\left(\left(\nabla_{X} J\right)\left(E_{s}\right),\left(\nabla_{J Y} J\right)\left(E_{s}\right)\right)\right),
$$

and by $(2.1)$ we get

$$
8 \pi \gamma_{1}(X, Y)=-4 \rho^{*}(X, J Y)-\sum_{s} g\left(\left(\nabla_{X} J\right)\left(E_{s}\right),\left(\nabla_{J Y} J\right)\left(E_{s}\right)\right) .
$$


Note that when $M$ is compact the 2-form $\gamma_{1}$ represents the first Chern class of $M$ in the de Rham cohomology group.

The Riemannian metric $g$ induces a metric on the bundle $\wedge^{2} T M$ of 2 vectors by $\left\langle X_{1} \wedge X_{2}, X_{3} \wedge X_{4}\right\rangle=\operatorname{det}\left(g\left(X_{i}, X_{j}\right)\right)$. The curvature operator $\mathscr{R}$ is the selfadjoint endomorphism of $\wedge^{2} T M$ defined by $\langle\mathscr{R}(X \wedge Y), Z \wedge W\rangle=$ $R(X, Y, Z, W)$. The Hodge star operator defines an endomorphism $*$ of $\bigwedge^{2} T M$ with $*^{2}=$ Id . Hence $\bigwedge^{2} T M=\bigwedge_{+}^{2} T M \oplus \bigwedge_{-}^{2} T M$, where $\bigwedge_{ \pm}^{2} T M$ are the subbundles of $\wedge^{2} T M$ corresponding to the $( \pm 1)$-eigenvalues of $*$. The block-decomposition of $\mathscr{R}$ with respect to the above splitting of $\wedge^{2} T M$ is

$$
\mathscr{R}=\left[\begin{array}{cc}
\tau / 12 \cdot \mathrm{Id}+\mathscr{W}_{+} & \mathscr{B} \\
{ }^{\mathscr{B}} & \tau / 12 \cdot \mathrm{Id}+\mathscr{W}_{-}
\end{array}\right]
$$

where $\tau$ is the scalar curvature; $\mathscr{B}$ and $\mathscr{W}=\mathscr{W}_{+}+\mathscr{W}_{-}$represent the traceless Ricci tensor and the Weyl conformal tensor, respectively. Recall that $M$ is called (anti-) self-dual if $\left(\mathscr{W}_{+}\right) \mathscr{W}_{-}=0$.

\section{CONFORMAlly KäHLER $*$-EINSTEIN SURFACES}

Recall [V] that a Hermitian surface $(M, J, g)$ is called (globally) conformally Kähler if the metric $g$ is conformal to a Kähler metric with respect to $J$.

Theorem 3.1. Any compact Hermitian *-Einstein surface with positive *-scalar curvature is conformally Kähler.

Proof. We first prove the following lemma.

Lemma 3.2. Any compact Hermitian *-Einstein surface is locally conformally Kähler.

Proof of the lemma. Let $M=(M, J, g)$ be a compact Hermitian *-Einstein surface with Lee form $\omega$ and Riemannian curvature tensor $R$. For any orthonormal $J$-frame $\left\{E_{s}\right\}$ (i.e., $J E_{1}=E_{2}, J E_{2}=-E_{1}, J E_{3}=E_{4}, J E_{4}=$ $\left.-E_{3}\right)$ we set

$$
\begin{array}{ll}
F_{1}=\left(E_{1}-i E_{2}\right) / \sqrt{2}, & F_{1}=\left(E_{1}+i E_{2}\right) / \sqrt{2}, \\
F_{2}=\left(E_{3}-i E_{4}\right) / \sqrt{2}, & F_{\overline{2}}=\left(E_{3}+i E_{4}\right) / \sqrt{2},
\end{array}
$$

and $K_{a b c d}=R\left(F_{a}, F_{b}, F_{c}, F_{d}\right)$, where the curvature tensor $R$ is continued by complex linearity. Then a direct computation involving (2.1) shows that $M$ is *-Einstein iff

$$
K_{121 \overline{1}}+K_{122 \overline{2}}=0, \quad K_{1 \overline{2} 1 \overline{1}}+K_{1 \overline{2} 2 \overline{2}}=0, \quad K_{1 \overline{1} 1 \overline{1}}-K_{2 \overline{2} 2 \overline{2}}=0 .
$$

The first identity of (3.1) together with a result of Kodâ [K, Lemma 6.3] implies that $d \omega$ is an anti-self-dual 2-form. Hence $d \omega=0$ since $M$ is compact.

Now we are ready to prove the theorem. Let $M$ be a Hermitian *-Einstein surface with $\tau^{*}>0$ everywhere on $M$. Consider the Riemannian metric $g^{*}$ on $M$ defined by

$$
g^{*}(X, Y)=\tau^{*} g(X, Y)+\sum_{s} g\left(\left(\nabla_{X} J\right)\left(E_{S}\right),\left(\nabla_{Y} J\right)\left(E_{S}\right)\right)
$$


where $\left\{E_{s}\right\}$ is an orthonormal frame. It follows easily that the metric $g^{*}$ is compatible with the complex structure $J$ since for any Hermitian manifold we have $\left(\nabla_{J X} J\right)(Y)=J\left(\nabla_{X} J\right)(Y)$ (cf., e.g., [Gr]). From (3.2), (2.4), and the fact that $M$ is a $*$-Einstein surface we deduce that the Kähler form $\Omega^{*}$ of $\left(M, J, g^{*}\right)$ is given by $\Omega^{*}=-8 \pi \gamma_{1}$, where $\gamma_{1}$ is the generalized first Chern form of $M$. Hence $d \Omega^{*}=0$, which shows that $g^{*}$ is a Kähler metric on $M$ with respect to $J$. In particular, the first Betti number of $M$ is even, and the theorem follows by a result of Vaisman [V].

Remarks. (1) From (2.4) it follows that the first Chern class of $M$ is positive, i.e., $M$ is an algebraic surface. This is another way to prove the existence of a Kähler metric on $M$.

(2) By the classification of compact complex surfaces with positive first Chern class (cf., e.g., [B, 11.13]) it follows that the only surfaces on which the existence of $*$-Einstein metrics with positive $*$-scalar curvature can be expected are $C P^{1} \times$ $C P^{1}$ and $\Sigma_{r}, 0 \leq r \leq 8$.

Theorem 3.1 reduces the problem of describing the compact Hermitian *Einstein surfaces with positive $*$-scalar curvature to the problem when the conformal class of a Kähler metric contains a $*$-Einstein metric. The next theorem gives a partial answer to this question.

Theorem 3.3. Let $M$ be a compact Kähler surface with Kähler form $\Omega$, Ricci form $\gamma$, and scalar curvature $\tau$. Let $F>0$ be a smooth function on $M$. Then

(i) the metric $\tilde{g}=F^{-2} \cdot g$ is $*$-Einstein iff

$$
(\tau / 4-\Delta F / F) \cdot \Omega=\gamma-2 i \partial \bar{\partial} F / F,
$$

where $\Delta$ is the Laplace operator. Moreover, the *-scalar curvature of $\tilde{g}$ is given by

$$
\tilde{\tau}^{*}=F^{2} \cdot \tau-2 F \Delta F-4\|d F\|^{2} .
$$

(ii) The conformal class of $g$ contains at most one (up to a constant) *Einstein metric.

Proof. To prove (i) we continue all tensor fields on $M$ by complex linearity. For a tensor field $Z$ of type $(0,2)$ we denote by $Z^{1,1}$ its $(1,1)$-part given by $Z^{1,1}(X, Y)=\frac{1}{2}(Z(X, Y)+Z(J X, J Y))$ for all complex vector fields $X, Y$ on $M$. Let $\rho_{0}\left(\tilde{\rho}_{0}\right)$ and $\rho_{0}^{*}\left(\tilde{\rho}_{0}^{*}\right)$ be the traceless Ricci tensor and the traceless $*$-Ricci tensor of the metric $g(\tilde{g})$, respectively.

Lemma 3.4. The metric $\tilde{g}$ is $*$-Einstein iff $\tilde{\rho}_{0}^{1,1}=0$.

Proof of the lemma. Denote by $\omega$ the 1 -form on $M$ defined by $\omega=d\left(\log F^{2}\right)$. It is well known (cf., e.g., [O2]) that the $*$-Ricci tensors of the metrics $g$ and $\tilde{g}$ are related by

$$
\tilde{\rho}^{*}(X, Y)=\rho^{*}(X, Y)+1 / 2(P(X, Y)+P(J X, J Y))
$$

for $X, Y \in \chi(M)$, where

$$
P(X, Y)=\left(\nabla_{X} \omega\right)(Y)+1 / 2 \cdot \omega(X) \omega(Y)-1 / 4 \cdot\|\omega\|^{2} \cdot g(X, Y) .
$$

Moreover,

$$
F^{2} \tilde{\tau}^{*}=\tau^{*}-\delta \omega-1 / 2 \cdot\|\omega\|^{2} .
$$


Assume that $\tilde{g}$ is a $*$-Einstein metric. Then $\left(\tilde{\rho}_{0}^{*}\right)^{1,1}=0$, and (2.2) shows that $\left(\tilde{\rho}_{0}\right)^{1,1}=0$. Conversely, let $\left(\tilde{\rho}_{0}\right)^{1,1}=0$. Then from $(2.2)$ it follows that $\left(\tilde{\rho}_{0}^{*}\right)^{1,1}=0$. On the other hand from (3.5) and the fact that $g$ is a Kähler metric (i.e., $\left.\rho=\rho^{*}\right)$ we get $\tilde{\rho}_{0}^{*}(X, Y)=\tilde{\rho}_{0}^{*}(J X, J Y)$. Hence $\tilde{\rho}_{0}^{*}=0$, which simply means that $\tilde{g}$ is a $*$-Einstein metric.

To prove statement (i) we first recall that the traceless Ricci tensors of the metrics $g$ and $\tilde{g}=F^{-2} g$ are related by (cf. [B, (1.161b)])

$$
\tilde{\rho}_{0}=2 / F \cdot(D d F+\Delta F / F \cdot g)+\rho_{0}
$$

Hence the first part of (i) follows from (3.8) and Lemma 3.4 using the fact that for Kähler manifolds the following identities hold:

$$
(D d F)^{1,1}(X, J Y)=-i(\partial \bar{\partial} F)(X, Y), \quad\left(\rho_{0}\right)^{1,1}=\rho_{0} .
$$

Formula (3.4) is an easy consequence of (3.7).

To prove (ii) let $F$ and $H$ be positive smooth functions on $M$ such that the metrics $F^{-2} g$ and $H^{-2} g$ are both *-Einstein. Then (3.3) implies $(H \Delta F-F \Delta H) \Omega=2 i(H \partial \bar{\partial} F-F \partial \bar{\partial} H)$, and taking into account the fact that the 2-forms $\Omega$ and $H \partial \bar{\partial} F-F \partial \bar{\partial} H$ are $\partial \bar{\partial}$-closed, we get $\partial \bar{\partial}(H \Delta F-F \Delta H) \wedge \Omega=$ 0 . On the other hand,

$$
\begin{aligned}
\Delta(H \Delta F-F \Delta H) \cdot d M & =2 i\langle\partial \bar{\partial}(H \Delta F-F \Delta H), \Omega\rangle \cdot d M \\
& =2 i \partial \bar{\partial}(H \Delta F-F \Delta H) \wedge \Omega
\end{aligned}
$$

since $* \Omega=\Omega$. Hence $\Delta(H \Delta F-F \Delta H)=0$, which implies $(H \Delta F-F \Delta H) \equiv$ const, since $M$ is compact. Now integrating over $M$ gives

$$
(H \Delta F-F \Delta H)=0 \text {. }
$$

An easy computation involving the definition of the Laplace operator shows that $\Delta(F / H)=2 \cdot\langle d(F / H), d(\log H)\rangle+1 / h^{2} \cdot(H \Delta F-F \Delta H)$. This, together with (3.9), implies that $\Delta U-2 \cdot\langle d U, d(\log H)\rangle=0$, where $U=F / H$. Now by the maximum principle for second order strongly elliptic differential operators we conclude that $U \equiv$ const, and statement (ii) is proved.

An immediate consequence of Theorem 3.3(ii) is the following.

Corollary 3.4. Let $(M, J, g)$ be a Kähler-Einstein surface. Then the only *Einstein metrics in the conformal class of $g$ are the constant multiples of $g$.

\section{Proof of Theorem 1.1 AND applications}

We begin the proof of Theorem 1.1 with the following

Lemma 4.1. A compact Hermitian Einstein surface $M$ is *-Einstein iff it is locally conformally Kähler.

Proof of the lemma. Using the same notation as in the proof of Lemma 3.2 we check easily that $M$ is Einstein iff

$$
\begin{array}{ll}
K_{121 \overline{2}}=K_{12 \overline{1} 2}=0, & K_{1 \overline{1} 1 \overline{1}}=K_{2 \overline{2} 2 \overline{2}}, \\
K_{1 \overline{2} 1 \overline{1}}+K_{1 \overline{2} 2 \overline{2}}=0, & K_{121 \overline{1}}=K_{122 \overline{2}} .
\end{array}
$$

On the other hand by [K, Lemma 6.3] and the fact that $M$ is compact it follows that $M$ is locally conformally Kähler iff

$$
K_{121 \overline{1}}+K_{122 \overline{2}}=0 \text {. }
$$

Hence the lemma follows from (3.1), (4.1), and (4.2). 
Now let $M$ be a compact Hermitian Einstein and $*$-Einstein surface. Then by a result of Derdzinski [D, Proposition 5] it follows that either $\mathscr{W}_{+}=0$ identically or $\mathscr{W}_{+} \neq 0$ everywhere on $M$. Consider the case $\mathscr{W}_{+} \equiv 0$. Then by [K] we get $\tau=3 \tau^{*}$, i.e., $\tau^{*}$ is a constant. Assume that $\tau^{*}<0$. Then a result of Sekigawa [S2] says that $M$ is a Kähler surface. Hence $\tau=\tau^{*}=0$, which is a contradiction. If $\tau^{*}=0$ then $\tau=0$ and (2.3) implies $2 \delta \omega+\|\left.\omega\right|^{2}=0$. Integrating over $M$ gives $\int_{M}\|\omega\|^{2} d M=0$, which shows that $M$ is a Kähler surface. It remains to consider the case $\tau^{*}>0$. Then by Theorem 3.1 it follows that $M$ is conformally Kähler, i.e., $\omega=d F$, where $F$ is a positive smooth function on $M$. Using (2.3) and $\tau=3 \tau^{*}$ we get

$$
2 \tau^{*}=2 \Delta F+\|d F\|^{2} .
$$

Let $F\left(p_{0}\right)=\min _{p \in M} F(p)$. Then $(d F)_{p_{0}}=0,(\Delta F)_{p_{0}} \leq 0$, and 4.3 implies $\tau^{*} \leq 0$, which is a contradiction. The arguments above show that if $\mathscr{W}_{+}=0$ then $M$ is a Ricci-flat Kähler surface.

Now consider the case when $\mathscr{W}_{+} \neq 0$ everywhere on $M$. We shall show that $\left\|\mathscr{W}_{+}\right\|^{2 / 3} \cdot g$ is a Kähler metric on $M$. By Lemma 4.2 we know that $M$ is locally conformally Kähler. This means that for any point $p \in M$ there exist a connected neighbourhood $U$ of $p$ and a positive smooth function $F$ on $U$ such that $F \cdot g$ is a Kähler metric with respect to $J$ on $U$. Since $M$ is Einstein by a result of Derdzinski [D, Proposition 5 (ii)], it follows that there is a constant $C_{U}$ such that $F=C_{U}\left\|\mathscr{W}_{+}\right\|^{2 / 3}$. Hence $\left\|\mathscr{W}_{+}\right\|^{2 / 3} \cdot g$ is a Kähler metric on $M$. Suppose that the tensor field $\mathscr{W}_{+}$is parallel. Then from [D, Proposition 5 (iii)] it follows that $\left\|\mathscr{W}_{+}\right\|^{2 / 3}$ is a constant, i.e., $g$ is a Kähler metric. It remains to consider the case when $\mathscr{W}_{+}$is not parallel. Now from [D, Theorem 2(ii)] we deduce that $\tilde{g}=\left\|\mathscr{W}_{+}\right\|^{2 / 3} \cdot g$ is an extremal Kähler metric whose scalar curvature $\tilde{\tau}$ is nonconstant and positive everywhere on $M$. Moreover

$$
\tau=\tilde{\tau}^{3}-6 \tilde{\tau} \tilde{\Delta} \tilde{\tau}-12 \tilde{g}(d \tilde{\tau}, d \tilde{\tau})>0 .
$$

On the other hand, from (3.4) it follows that

$$
\tau^{*}=\tilde{\tau}^{3}-2 \tilde{\tau} \tilde{\Delta} \tilde{\tau}-4 \tilde{g}(d \tilde{\tau}, d \tilde{\tau}) .
$$

Hence $3 \tau^{*}=2 \tilde{\tau}^{3}+\tau>0$, and statement (ii) is proved. The converse statement follows by Lemma 4.2 and the results of Derdzinski [D].

From Theorem 4.1 we obtain the following Hermitian analogs of the result of Sekigawa and Vanhecke [SV] mentioned in the introduction.

Corollary 4.2. Any compact Hermitian Einstein and $*$-Einstein surface with constant *-scalar curvature is Kählerian.

Corollary 4.3. Any compact Hermitian anti-self-dual and $*$-Einstein surface with constant $*$-scalar curvature is Kählerian and Ricci-flat.

Proof. From (2.2) it follows that the Ricci tensor $\rho$ satisfies the following identity: $\rho(X, Y)+\rho(J X, J Y)=\tau / 2 \cdot g(X, Y)$ for all $X, Y \in \chi(M)$. This, together with (2.5) and an integral formula of Sekigawa [S2, (3.29)], gives

$$
\int_{M}\left(\left\|\mathscr{W}_{+}\right\|^{2}+\tau^{2} / 48-\left(\tau^{*}\right)^{2} / 16-\|\mathscr{B}\|^{2}\right) d M=1 / 16 \cdot \int_{M} \tau^{*}\|\omega\|^{2} d M .
$$


On the other hand, $\tau=3 \tau^{*}$ since $\mathscr{W}_{+}=0$ (cf. [K]). Hence from (2.3), (4.4), and the fact that $\tau^{*}$ is a constant we get $\int_{M}\|\mathscr{B}\|^{2} d M=0$. Therefore, $g$ is an Einstein metric, and the corollary follows by [K] and Corollary 4.2.

\section{REFERENCES}

[B] A. L. Besse, Einstein manifolds, Springer-Verlag, New York, 1987.

[D] A. Derdzinski, Self-dual Kähler manifolds and Einstein manifolds of dimension four, Compositio Math. 49 (1983), 405-433.

[G0] S. I. Goldberg, Integrability of almost Kähler manifolds, Proc. Amer. Math. Soc. 21 (1969), 96-100.

[Gr] A. Gray, Curvature identities for Hermitian and almost Hermitian manifolds, Tôhoku Math. J. 28 (1976), 601-612.

[K] T. Kodâ, Self-Dual and anti-self-dual Hermitian surfaces, Kodai Math. J. 10 (1987), 335342.

[O1] Z. Olszak, A note on almost Kähler manifolds, Bull. Acad. Polon. Sci. Ser. Sci. Math. Astronom. Phys. 26 (1978), 139-141.

[O2] $\quad-$ On the existence of generalized complex space forms, Israel J. Math. 65 (1989), 214218.

[S1] K. Sekigawa, On some compact Einstein almost Kähler manifolds, J. Math. Soc. Japan 39 (1987), 677-684.

[S2] _ _ On some 4-dimensional compact almost Hermitian manifolds, J. Ramanujan Math. Soc. 2 (1987), 101-116.

[SV] K. Sekigawa and L. Vanhecke, Four-dimensional almost Kähler Einstein manifolds, Ann. Math. Pura Appl. (4) 157 (1990), 149-160.

[TV] F. Tricceri and L. Vanhecke, Curvature tensors on almost Hermitian manifolds, Trans. Amer. Math. Soc. 267 (1981), 365-398.

[V] I. Vaisman, On locally and globally conformal Kähler manifolds, Trans. Amer. Math. Soc. 262 (1980), 533-542.

Institute of Mathematics, Bulgarian Academy of Sciences, ul. "Acad. G. BontcheV", BL.8, 1090 Sofia, Bulgaria 